\title{
Hindrance of spasticity after spinal cord injury
}

Spinal Cord (2015) 53, 711; doi:10.1038/sc.2015.46; published online 17 March 2015

Comment on: Spinal Cord (2015) 53, 663-667; doi:10.1038/ sc.2015.41; published online 17 March 2015

Spasticity may be a severe problem for patients with spinal cord injury (SCI). The authors aim to add knowledge to this clinical entity by presenting their findings derived from a questionnaire that was sent out to patients at four subsequent points in time during the first year after SCI.

To access the negative impact of spasticity on daily activities, the authors introduce a new type of evaluation criterion: hindrance. Detailed data are presented about the self-rating of patients how much they felt the hindrance induced by spasticity for a predefined set of activities. Very little additional information is given about the impairment of the patients, especially no clinical exam data (for example, sensory and motor scores from the International Standards for Neurological Classification of Spinal Cord Injury ${ }^{1}$ ) or data from validated assessments scales for spasticity (for example, the Modified Ashworth Scale ${ }^{2}$ or Spinal Cord Assessment Tool for Spastic Reflexes ${ }^{3}$ ) was performed. Few confounding factors are taken into account.

The lecture of the paper left us unhappy. The biggest problem results from the missing definition of 'hindrance' and 'spasticity'. Although it is actually an interesting approach to identify factors that actually 'hinder' patients beyond their disability due to neurological impairments, the authors cross a few lines that should be crossed with caution: a novel assessment should only be used in a clinical trial after validation. In this case, no information is provided on validity and reliability of the assessments, nor do we even know whether the English translation has the same meaning as the original Dutch word used in the questionnaire. This may lead to a potential bias in the results of the study. In the presented work, no validation of the assessment has been performed, and the definition of 'hindrance' and 'spasticity' is left mostly to the patient, which is especially worrisome because patients easily confuse spasticity with cramps or pain. The study is called a prospective cohort study, but the criteria for dichtomization were defined retrospectively according to the distribution of the assessed scores, for example, spasticity score. It is even not clear, why dichtomization is necessary.

Different number of data sets at different time points, which are not related to the onset of SCI but rather to vague phases in rehabilitation, are analyzed, which may mask differences. Therefore, the conclusion of the paper cannot be drawn on the basis of the applied methodology.

All in all, we agree with the authors: more research is needed to tackle this subject in particular by using internationally accepted and validated assessments (http://www.scireproject.com/outcomemeasures), starting with a common definition of 'spasticity' and 'hindrance' to allow for its quantification.

\section{CONFLICT OF INTEREST}

The author declares no conflict of interest.

$\mathrm{R} \mathrm{Abel}^{1}$ and R Rupp ${ }^{2}$

${ }^{1}$ Klinikum Bayreuth Klinik für Querschnittgelähmte, Hohe Warte, Bayreuth, Germany and ${ }^{2}$ Klinik für Paraplegiologie, Universitätsklinikum Heidelberg, Experimentelle Neurorehabilitation, Schlierbacher Landstrasse, Heidelberg, Germany E-mail: abel_rainer@hotmail.com

1 Kirshblum S, Waring W 3rd. Updates for the International Standards for Neurological Classification of Spinal Cord Injury. Phys Med Rehabil Clin N Am 2014; 25: 505-517.

2 Bohannon RW, Smith MB. Interrater reliability of a modified Ashworth scale of muscle spasticity. Phys Ther 1987; 67: 206-207.

3 Benz EN, Hornby TG, Bode RK, Scheidt RA, Schmit BD. A physiologically based clinical measure for spastic reflexes in spinal cord injury. Arch Phys Med Rehabil 2005; 86: 52-59. 